\section{Severity of hyperechoic pancreas on ultrasonography as a risk factor for glycemic progression}

\author{
Jiyun Oh${ }^{1}$, Hyun Jeong Park', Eun Sun Lee ${ }^{1}$, Sung Bin Park', Byung Ihn Choi', \\ Soohyun $\mathrm{Ahn}^{2}$
}

'Department of Radiology, Chung-Ang University Hospital, Chung-Ang University College of Medicine, Seoul; ${ }^{2}$ Department of Mathematics, Ajou University, Suwon, Korea

Purpose: The aim of this study was to evaluate the association between the severity of hyperechoic pancreas (HP) on ultrasonography (US) and glycemic progression.

Methods: In total, 1,386 participants who underwent abdominal US as part of health examinations between December 2008 and May 2014 were included in this retrospective study. We classified pancreatic echogenicity on a 4-point scale, and compared it using two distinct criteria: fatty pancreas (FP) 1 criterion (normal vs. $\geq$ mild HP) and FP2 criterion (normal/mild HP vs. $\geq$ moderate HP). According to the presence of nonalcoholic fatty liver disease (NAFLD), participants were subdivided into four groups: non-NAFLD and non-HP, isolated NAFLD, isolated HP, and HP with NAFLD. Glycemic progression was defined as progression from normoglycemia to prediabetes or diabetes or progression from prediabetes to diabetes.

Results: During the follow-up (median, 5.9 years), 262 of the 1,386 participants developed glycemic progression. Using FP2, the probability of glycemic progression across the four subgroups showed cumulative aggravation for NAFLD and HP (all $P<0.05$ ). Isolated HP showed a higher probability of glycemic progression than isolated NAFLD according to FP2 $(P<0.001)$. The highest probability of glycemic progression was observed in patients with both NAFLD and $\mathrm{HP}(\mathrm{P}<0.001)$. The hazard ratio for glycemic progression increased with the severity of HP.

Conclusion: Increasing severity of HP on US was found to be significantly correlated with glycemic progression. Moreover, isolated HP of moderate or greater severity predicted glycemic progression independent of NAFLD.

Keywords: Ultrasonography; Hyperechoic pancreas; Fatty pancreas; Diabetes;

Nonalcoholic fatty liver disease

\section{Introduction}

Increased levels of triglyceride and free fatty acids cause ectopic fat depositions in the liver, heart, muscles, and pancreas in a condition called steatosis. Ectopic fat accumulation in the liver, termed nonalcoholic fatty liver disease (NAFLD), is associated with an increased risk of diabetes, metabolic syndrome, and cardiovascular disease [1-4]. Similar to this condition, ectopic fat deposition in

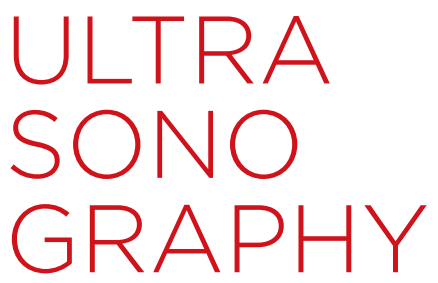

ORIGINAL ARTICLE

https://doi.org/10.14366/usg.20122 pISSN: 2288-5919 - elSSN: 2288-5943 Ultrasonography 2021;40:499-511

Received: August 10, 2020

Revised: January 6, 2021

Accepted: January 16, 2021

Correspondence to: Hyun Jeong Park, MD, Department of Radiology, Chung-Ang University Hospital, Chung-Ang University College of Medicine, 102 Heukseok-ro, Dongjak-gu, Seoul 06973, Korea

Tel. +82-2-6299-3199

Fax. +82-2-6299-2017

E-mail: seolly1024@cau.ac.kr

This is an Open Access article distributed under the terms of the Creative Commons Attribution NonCommercial License (http://creativecommons.org/ licenses/by-nc/4.0// which permits unrestricted noncommercial use, distribution, and reproduction in any medium, provided the original work is properly cited.

Copyright (C) 2021 Korean Society of Ultrasound in Medicine (KSUM)

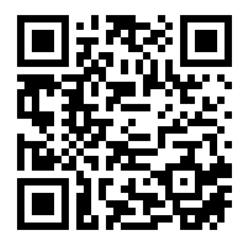

How to cite this article: Oh J, Park HJ, Lee ES, Park SB, Choi BI, Ahn S. Severity of hyperechoic pancreas on ultrasonography as a risk factor for glycemic progression. Ultrasonography. 2021 Oct;40(4):499-511. 
the pancreas is known as pancreatic steatosis or fatty pancreas, which has recently received increasing attention. Although many studies have revealed that fatty pancreas is also associated with an increased risk of type 2 diabetes, NAFLD, and metabolic risk factors, the association is not fully understood [5-11].

Ultrasonography (US) has traditionally been used to evaluate fat accumulation in the pancreas $[12,13]$. However, there is neither a widely accepted modality nor a cutoff point for diagnosing fatty pancreas [14]. Fatty pancreas is defined as a hyperechoic pancreas (HP) on US compared with the echogenicity of the liver or renal cortex $[6,8,15]$. Although a few studies have been reported with a small number of selected participants $[10,16,17]$, there have been no large-scale, longitudinal studies focusing on the severity of fatty pancreas and glycemic progression. Additionally, the diagnostic criteria are slightly vague and can be occasionally challenging with respect to the possible coexistence of NAFLD or a limited sonic window, especially in mild fatty pancreas.

Meanwhile, a strong association between NAFLD and fatty pancreas has been reported in previous studies [18-20]. The prevalence of this concomitant condition could be up to $50 \%$ in both Western and Asian countries, and can most likely be attributed to the similar pathogenesis associated with metabolic risk factors. However, data are limited on the association of isolated fatty pancreas with glycemic progression independent of NAFLD and its synergistic effect on the progression of diabetes.

In daily practice, we encounter HP of varying severity with or without concomitant NAFLD, diabetes, or other cardiometabolic risk factors. However, whether the severity of HP is independently associated with future glycemic progression is unclear. Moreover, whether the severity of HP is associated with the aforementioned diseases independently of NAFLD remains largely unknown.

Therefore, this study aimed to evaluate the association between the severity of HP on US and glycemic progression with the influence of concomitant NAFLD.

\section{Materials and Methods}

\section{Compliance with Ethical Standards}

This retrospective study was approved by the institutional review board Chung-Ang University Hospital in Korea (1811-016-16223), which waived the requirement for informed consent owing to the retrospective study design.

\section{Sample Size}

In a previous study [17], the prevalence of HP on US was around $15 \%(147+326 / 8,856=15.43 \%)$. The hazard ratio (HR) for glycemic progression in participants with HP on US was 1.54. On the basis of the HR of 1.54 and $80 \%$ power with a $5 \%$ significance level, the minimum sample size for estimating glycemic progression was calculated to be 261. Additionally, considering the development of diabetes or prediabetes on follow-up ( $5 \%$ and $14 \%$, respectively), the minimum total number of samples was determined to be 1,383 .

\section{Participants}

Between January 2008 and December 2014, we analyzed individuals who underwent US for health screening at a tertiary hospital. Because a substantial proportion of these individuals regularly attended health examinations, those who had undergone a follow-up examination at least 2 years after the baseline examination were included in this study. We excluded individuals who met the following criteria: (1) aged $<20$ years, (2) estimated alcohol consumption $>20 \mathrm{~g} /$ day, (3) a history of viral hepatitis or seropositive for hepatitis B and C virus antigen, (4) a history of liver or pancreas surgery or medical renal disease, (5) use of diabetes medications or a diagnosis of diabetes, and (6) incomplete laboratory data from the initial and/or follow-up examinations. The flow diagram of this study is shown in Fig. 1.

\section{Clinical and Laboratory Parameters}

Data on age, sex, comorbidities including diabetes, alcohol consumption, and medications used were collected. All participants underwent height and body weight measurements while wearing light clothing to calculate the body mass index $\left(\mathrm{BMI}\right.$, in $\left.\mathrm{kg} / \mathrm{m}^{2}\right)$. For waist circumference, we used a tape measure and measured in the middle part between the lowest rib and the iliac crest of the pelvis, horizontal to the ground with the participant in an upright position. Systolic and diastolic blood pressures were recorded in the sitting position.

After overnight fasting, all participants underwent blood tests including fasting blood glucose, plasma hemoglobin A1c (HbA1c), total cholesterol, triglyceride, high-density lipoprotein, low-density lipoprotein, blood urea nitrogen, and creatinine tests.

Metabolic syndrome was defined according to the updated National Cholesterol Education Program Adult Treatment Panel III and the abdominal obesity criteria of the Korean Society for the Study of Obesity. Diabetes was diagnosed according to the American Diabetes Association 2014 recommendations (HbA1C $\geq 6.5 \%$, fasting glucose level $\geq 126 \mathrm{mg} / \mathrm{dL}$, or 2-hour postprandial plasma glucose level $\geq 120 \mathrm{mg} / \mathrm{dL}$ ). Prediabetes was diagnosed according to the American Diabetes Association 2014 recommendations (fasting glucose level, 100-125 mg/dL; 2-hour postprandial glucose level, $140-199 \mathrm{mg} / \mathrm{dL} ;$ or HbA1C, $5.7 \%-6.4 \%$ ).

Glycemic progression was measured as an endpoint in our followup analysis and was defined as progression from normoglycemia to 
4,697 Participants with baseline examination including abdominal US

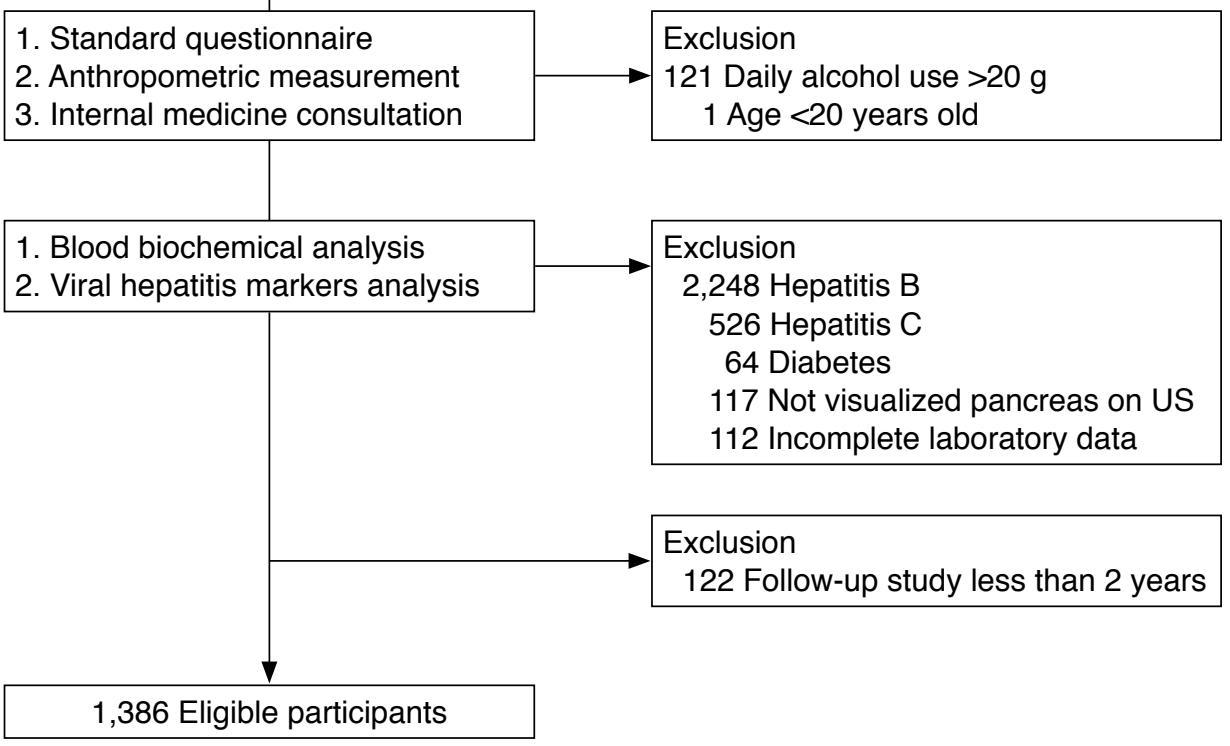

Fig. 1. Flow diagram showing the patient selection criteria and study flow. US, ultrasonography. prediabetes or diabetes or progression from prediabetes to diabetes.

\section{Analysis of Abdominal US Images}

After overnight fasting, the participants underwent abdominal US, performed by designated board-certified radiologists using a 3.5MHz convex-array probe (LOGIQ7, GE Healthcare, Milwaukee, WI, USA). The radiologists were blinded to the laboratory findings.

To grade NAFLD and HP, two radiologists (J.O. and H.J.P., with 3 and 14 years of experience in abdominal US, respectively) independently reviewed abdominal US images on a commercial workstation with a 2,000 $\times 2,000$ picture archiving and communication system monitor (Centricity, GE Healthcare). Both radiologists were blinded to other clinical and laboratory parameters. In cases of disagreement, a third observer (E.S.L. with 14 years of experience in abdominal US) was consulted to reach a consensus.

The US diagnostic criteria for NAFLD were increased parenchymal echogenicity compared with the right renal cortex as well as blurring of the hepatic vasculature and an echogenic diaphragm. Liver echogenicity was graded using a 4-point scale according to the criteria described by Needleman et al. [21] as follows: (1) normal, equal to, or slightly greater echogenicity than that of the renal cortex; (2) mild, diffusely increased hepatic echogenicity, but periportal and diaphragmatic echogenicity is still appreciable; (3) moderate, diffusely increased hepatic echogenicity obscuring periportal echogenicity, but diaphragmatic echogenicity is still appreciable; (4) severe, diffusely increased hepatic echogenicity obscuring periportal as well as diaphragmatic echogenicity.
Compared with the normal echogenicity of the pancreas, which is similar to the normal liver or kidney parenchymal echogenicity, the US diagnostic criterion for HP was higher echogenicity in the pancreas than that in the left lobe of the liver under the same US window. If the left hepatic lobe was not displayed in the same window for a direct comparison or showed increased echogenicity, we compared the echogenicity of the pancreas with that of the right kidney and spleen indirectly under two parallel US windows as described by Choi et al. [6] and Al-Haddad et al. [18]. Pancreatic echogenicity was graded using a 4-point scale, comparing with the echogenicity of the adjacent left hepatic lobe and retroperitoneal fat: (1) normal, similar echogenicity to the liver; (2) mild, slightly higher echogenicity than the liver; (3) clearly higher echogenicity than the liver, but lower echogenicity than the retroperitoneal fat; and (4) similar or higher echogenicity compared with the retroperitoneal fat (Fig. 2).

\section{Statistical Analysis}

All continuous variables are expressed as mean \pm standard deviation. The study participants were divided into two groups based on glycemic status: the normal group and the glycemic progression group, defined as progression from normoglycemia to prediabetes or diabetes or progression from prediabetes to diabetes on follow-up. The interobserver agreements for the severity of NAFLD and HP on US between the two radiologists were analyzed using the $\mathrm{K}$ statistic and interpreted as follows: poor, $<0.20$; fair, 0.20-0.39; moderate, $0.40-0.59$; substantial, $0.60-0.79$; and almost perfect, $\geq 0.80$. 




A

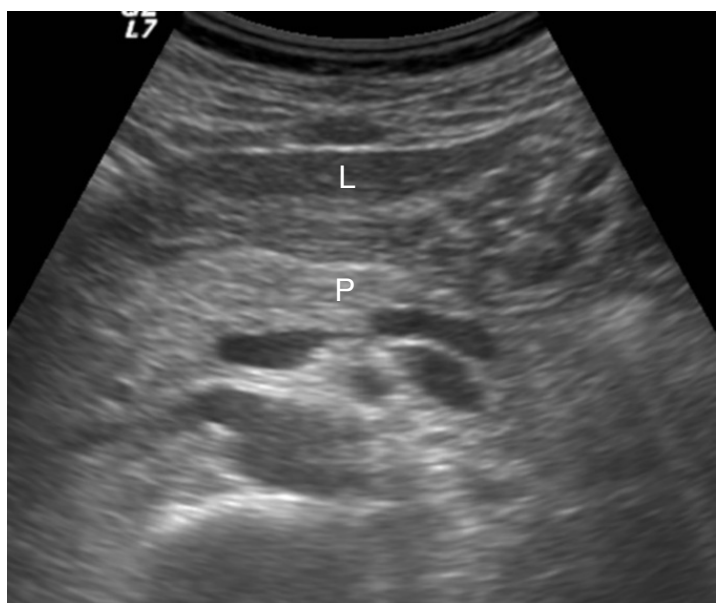

C

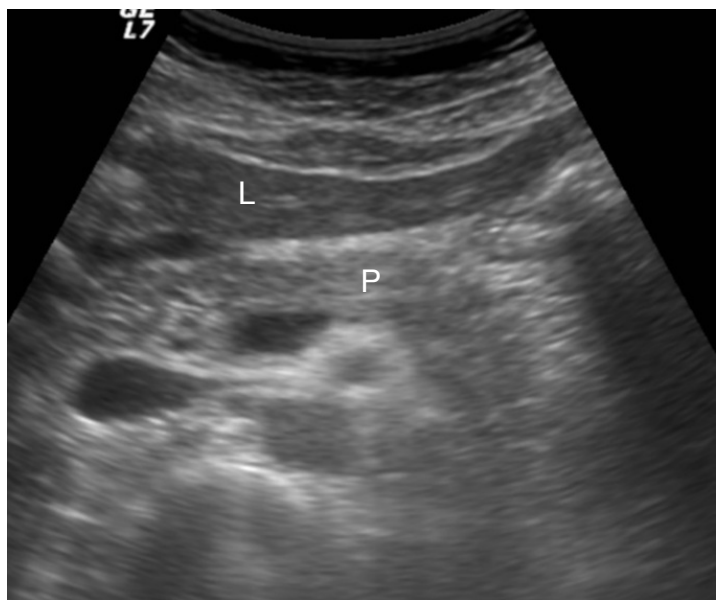

B

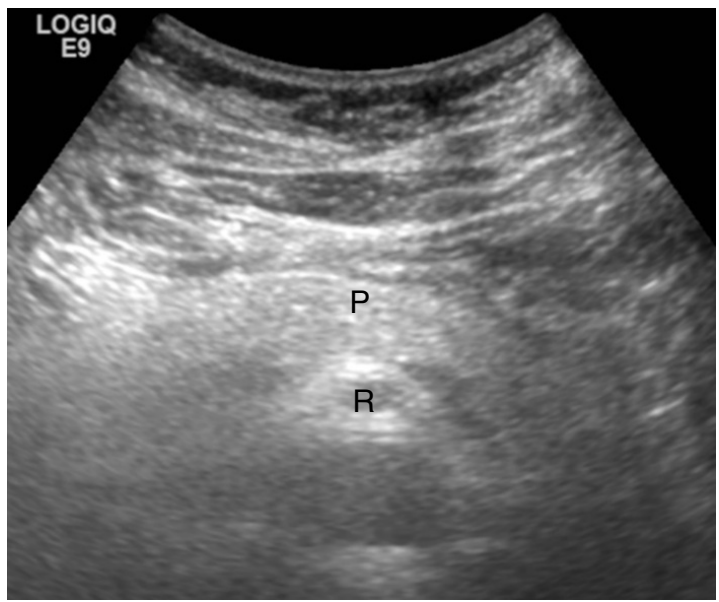

D

Fig. 2. Four-point scale of hyperechoic pancreas (HP).

A. The normal pancreas is defined as similar to the echogenicity than that of the left hemiliver. B. The mild HP is defined as slightly higher echogenicity than that of the left hemiliver. C. The moderate HP is defined as definite increase in echogenicity compared with that of the left hemiliver, but lower echogenicity than that of the retroperitoneal fat. D. The severe HP is defined as higher echogenicity than that of the retroperitoneal fat. L, liver; P, pancreas; $R$, retroperitoneal fat.

We divided HP using two criteria according to US severity for determining the optimal cutoff affecting glycemic progression as follows: the fatty pancreas 1 (FP1) criterion (normal vs. $\geq$ mild HP) and the fatty pancreas 2 (FP2) criterion (normal and mild HP vs. moderate and severe HP). On the basis of the above criteria, the participants were subdivided into four groups according to the presence of NAFLD and HP (FP1 and FP2) (Table 1). Continuous variables among the four study groups were compared using analysis of variance. The Fisher exact test or the chi-square test was used to analyze differences in categorical variables among groups. To identify factors related to glycemic progression controlling for confounding factors, univariable and multivariable Cox proportional hazard regression models were applied. Additionally, to identify
Table 1. Summary of abbreviations and diagnostic criteria

\begin{tabular}{|c|c|}
\hline Abbreviations of groups & Diagnostic criteria \\
\hline \multicolumn{2}{|l|}{ HP criterion for dichotomization } \\
\hline FP 1 criterion & Normal vs. $\geq$ mild HP \\
\hline FP 2 criterion & $\begin{array}{l}\text { Normal and mild HP vs. moderate and } \\
\text { severe HP }\end{array}$ \\
\hline \multicolumn{2}{|l|}{ Four combination groups } \\
\hline Group 1 & Non-NAFLD and non-HP \\
\hline Group 2 & Isolated NAFLD \\
\hline Group 3 & Isolated HP \\
\hline Group 4 & HP with NAFLD \\
\hline
\end{tabular}

HP, hyperechoic pancreas; FP 1, fatty pancreas 1; FP 2, fatty pancreas 2; NAFLD, nonalcoholic fatty liver disease. 
independent associations of glycemic progression with HP and NAFLD, we analyzed groups 1 and 2 as reference groups.

Survival curves for glycemic progression were drawn according to the status of NAFLD, HP, or combination groups of NAFLD and HP. The cumulative incidence of glycemic progression was compared using the log-rank test between groups according to the status of NAFLD, HP, or their combinations. Additionally, to identify the relationship with glycemic progression according to the severity of HP on US, a Cox proportional hazard model was applied.

All statistical analyses were performed using $R$ version 3.5.0 (The R Foundation for Statistical Computing, Vienna, Austria). A P-value of $<0.05$ was considered to indicate statistical significance.

\section{Results}

\section{Clinical and Ultrasonographic Characteristics of the Study Participants}

In total, 1,386 participants were recruited for the database analysis (Fig. 1). Among them, 786 (56.7\%) were men. The mean age and
BMI were $47.2 \pm 10.6$ years and $23.5 \pm 3.2 \mathrm{~kg} / \mathrm{m}^{2}$, respectively, and 164 participants (11.8\%) had metabolic syndrome. At the initial examination, 1,099 participants (79.2\%) were normoglycemic and 287 participants $(20.6 \%)$ had prediabetes. The number of participants with normal, mild, moderate, and severe degrees of echogenicity on US was 855 (61.7\%), $283(20.4 \%), 186(13.4 \%)$, and $62(4.5 \%)$ in the liver and 417 (30.1\%), 405 (30.0\%), 368 (26.6\%), and $196(14.1 \%)$ in the pancreas, respectively. Among the participants with mild fatty pancreas, 30.9\% (125 of 405) showed concomitant NAFLD. The interobserver agreement for the severity of NAFLD and HP on US was almost perfect $(\mathrm{K}=0.85)$ and substantial ( $\mathrm{K}$ $=0.78)$, respectively.

\section{Follow-up Analysis of Glycemic Progression}

During the median follow-up period of 5.9 years (range, 2.0 to 10.4 years), 262 of the 1,386 participants developed glycemic progression (incidence rate, 189 per 1,000 person-years), including 19 participants who developed incident diabetes (incidence rate, 13.7 per 1,000 person-years).

Table 2. Demographic characteristics and biochemical data stratified according to the status of NAFLD and HP based on the FP1 criterion

\begin{tabular}{|c|c|c|c|c|c|}
\hline Characteristic & $\begin{array}{c}\text { Group } 1 \\
\text { No NAFLD or HP } \\
(n=397)\end{array}$ & $\begin{array}{c}\text { Group } 2 \\
\text { Isolated NAFLD } \\
(n=20)\end{array}$ & $\begin{array}{c}\text { Group } 3 \\
\text { Isolated HP } \\
(n=458)\end{array}$ & $\begin{array}{c}\text { Group } 4 \\
\text { NAFLD and HP } \\
(n=511)\end{array}$ & P-value \\
\hline Age (year) & $42.6 \pm 9.4$ & $45.1 \pm 8.8$ & $49.0 \pm 10.5$ & $48.5 \pm 10.5$ & $<0.001$ \\
\hline Male sex & $155(39.0)$ & $17(85.0)$ & $248(54.1)$ & $379(74.2)$ & $<0.001$ \\
\hline MS & $5(1.3)$ & $1(5.0)$ & $28(6.1)$ & $130(25.4)$ & $<0.001$ \\
\hline Weight (kg) & $57.8 \pm 9.4$ & $66.7 \pm 10.4$ & $63.4 \pm 10.3$ & $72.3 \pm 11.7$ & $<0.001$ \\
\hline $\operatorname{BMI}\left(\mathrm{kg} / \mathrm{m}^{2}\right)$ & $21.3 \pm 2.5$ & $23.6 \pm 2.0$ & $23.2 \pm 2.6$ & $25.6 \pm 3.0$ & $<0.001$ \\
\hline Waist circumference (cm) & $75.2 \pm 7.4$ & $83.8 \pm 6.8$ & $80.9 \pm 8.1$ & $88.6 \pm 7.9$ & $<0.001$ \\
\hline $\mathrm{SBP}(\mathrm{mm} \mathrm{Hg})$ & $114.0 \pm 12.0$ & $124.9 \pm 9.1$ & $120.1 \pm 13.6$ & $124.9 \pm 11.5$ & $<0.001$ \\
\hline $\mathrm{DBP}(\mathrm{mm} \mathrm{Hg})$ & $68.3 \pm 9.2$ & $76.7 \pm 6.2$ & $72.4 \pm 10.4$ & $75.6 \pm 9.4$ & $<0.001$ \\
\hline GLC & $88.0 \pm 9.6$ & $92.4 \pm 11.8$ & $93.5 \pm 7.5$ & $97.8 \pm 12.3$ & $<0.001$ \\
\hline $\mathrm{HbA1c}$ & $5.3 \pm 0.3$ & $5.3 \pm 0.4$ & $5.5 \pm 0.4$ & $5.7 \pm 0.4$ & $<0.001$ \\
\hline $\mathrm{TG}(\mathrm{mg} / \mathrm{dL})$ & $77.4 \pm 41.7$ & $154.4 \pm 52.3$ & $104.6 \pm 73.5$ & $150.8 \pm 83.7$ & $<0.001$ \\
\hline $\mathrm{Cr}(\mathrm{mg} / \mathrm{dL})$ & $0.9 \pm 0.2$ & $1.0 \pm 0.1$ & $1.0 \pm 0.2$ & $1.0 \pm 0.2$ & $<0.001$ \\
\hline BUN (mg/dL) & $12.9 \pm 3.4$ & $12.3 \pm 2.7$ & $13.6 \pm 3.5$ & $14.2 \pm 4.1$ & 0.001 \\
\hline LDL level & $115.8 \pm 28.0$ & $135.3 \pm 27.9$ & $124.4 \pm 32.6$ & $130.1 \pm 34.6$ & $<0.001$ \\
\hline HDL level & $57.1 \pm 11.4$ & $46.5 \pm 7.4$ & $53.4 \pm 11.5$ & $47.8 \pm 9.0$ & $<0.001$ \\
\hline Total cholesterol & $188.7 \pm 32.9$ & $212.7 \pm 33.8$ & $198.7 \pm 36.3$ & $207.6 \pm 38.4$ & $<0.001$ \\
\hline NAFLD severity (mild/moderate/severe) & - & $17(85.0) / 3(15.0) / 0$ & - & $266(52.1) / 183(35.8) / 62(12.1)$ & $<0.001$ \\
\hline HP severity (mild/moderate/severe) & - & - & $280(61.1) / 135(29.5) / 43(9.4)$ & $125(24.5) / 233(45.6) / 153(29.9)$ & $<0.001$ \\
\hline
\end{tabular}

Values are presented as mean \pm standard deviation or number (\%).

FP1 (fatty pancreas 1) criterion, normal vs. mild HP or more.

NAFLD, nonalcoholic fatty liver disease; HP, hyperechoic pancreas; MS, metabolic syndrome; BMI, body mass index; SBP, systolic blood pressure; DBP, diastolic blood pressure; GLC, fasting blood glucose; HbA1c, plasma hemoglobin A1c; TG, triglyceride; Cr, creatinine level; BUN, blood urea nitrogen; LDL, low-density lipoprotein; HDL, high-density lipoprotein. 
Table 3. Demographic characteristics and biochemical data stratified according to the status of NAFLD and HP based on the FP2 criterion

\begin{tabular}{|c|c|c|c|c|c|}
\hline Characteristic & $\begin{array}{c}\text { Group } 1 \\
\text { Non-NAFLD and non-HP } \\
(\mathrm{n}=677)\end{array}$ & $\begin{array}{c}\text { Group } 2 \\
\text { Isolated NAFLD } \\
(n=145)\end{array}$ & $\begin{array}{l}\text { Group } 3 \\
\text { Isolated HP } \\
(n=178)\end{array}$ & $\begin{array}{c}\text { Group } 4 \\
\text { HP with NAFLD } \\
(n=386)\end{array}$ & P-value \\
\hline Age (year) & $44.6 \pm 49.8$ & $46.4 \pm 10.0$ & $51.7 \pm 11.3$ & $49.1 \pm 10.5$ & $<0.001$ \\
\hline Male sex & $301(44.5)$ & $113(77.9)$ & $102(57.3)$ & $283(73.3)$ & $<0.001$ \\
\hline MS & $14(2.0)$ & $17(11.7)$ & $19(10.7)$ & $283(29.5)$ & $<0.001$ \\
\hline Weight (kg) & $59.5 \pm 9.8$ & $69.8 \pm 11.1$ & $65.8 \pm 10.5$ & $72.9 \pm 11.8$ & $<0.001$ \\
\hline BMI $\left(\mathrm{kg} / \mathrm{m}^{2}\right)$ & $21.9 \pm 2.6$ & $24.5 \pm 2.6$ & $24.0 \pm 2.6$ & $25.9 \pm 3.2$ & $<0.001$ \\
\hline Waist circumference (cm) & $77.1 \pm 7.7$ & $85.7 \pm 7.2$ & $83.0 \pm 8.9$ & $89.4 \pm 8.3$ & $<0.001$ \\
\hline $\mathrm{SBP}(\mathrm{mmHg})$ & $116.1 \pm 13.2$ & $123.3 \pm 10.7$ & $121.4 \pm 12.6$ & $125.5 \pm 11.6$ & $<0.001$ \\
\hline $\mathrm{DBP}(\mathrm{mmHg})$ & $69.8 \pm 10.1$ & $74.4 \pm 7.8$ & $73.2 \pm 10.1$ & $76.1 \pm 9.8$ & $<0.001$ \\
\hline $\mathrm{GLC}$ & $89.2 \pm 10.4$ & $93.5 \pm 9.6$ & $94.9 \pm 12.2$ & $99.1 \pm 12.7$ & $<0.001$ \\
\hline $\mathrm{HbA1c}$ & $5.4 \pm 0.4$ & $5.5 \pm 0.3$ & $5.6 \pm 0.4$ & $5.7 \pm 0.4$ & $<0.001$ \\
\hline $\mathrm{TG}(\mathrm{mg} / \mathrm{dL})$ & $86.1 \pm 61.1$ & $144.5 \pm 78.6$ & $114.1 \pm 61.9$ & $153.3 \pm 84.2$ & $<0.001$ \\
\hline $\mathrm{Cr}(\mathrm{mg} / \mathrm{dL})$ & $1.0 \pm 0.2$ & $1.1 \pm 0.3$ & $1.0 \pm 0.2$ & $1.0 \pm 0.2$ & $<0.001$ \\
\hline BUN (mg/dL) & $13.1 \pm 3.4$ & $14 \pm 5.4$ & $13.8 \pm 3.6$ & $14.1 \pm 3.4$ & $<0.001$ \\
\hline LDL level & $118.3 \pm 29.2$ & $128.5 \pm 34.5$ & $128.7 \pm 35.3$ & $130.9 \pm 34.4$ & $<0.001$ \\
\hline HDL level & $55.9 \pm 11.5$ & $47.9 \pm 7.6$ & $52.3 \pm 11.4$ & $47.7 \pm 9.4$ & $<0.001$ \\
\hline Total cholesterol & $191.6 \pm 33.3$ & $205.7 \pm 36.7$ & $203.5 \pm 40.2$ & $208.6 \pm 38.8$ & $<0.001$ \\
\hline NAFLD severity (mild/moderate/severe) & - & $\begin{array}{c}118(81.4) / 26(17.9) / \\
1(0.7)\end{array}$ & - & $\begin{array}{c}266(68.9) / 183(47.4) / \\
62(16.1)\end{array}$ & $<0.001$ \\
\hline HP severity (mild/moderate/severe) & $280(41.4) / 0 / 0$ & $125(86.2) / 0 / 0$ & $0 / 135(75.8) / 43(24.2)$ & 0 /233 (60.4)/153 (39.6) & $<0.001$ \\
\hline
\end{tabular}

Values are presented as means \pm standard deviation or number (\%).

FP2 (fatty pancreas 2) criterion, normal and mild HP vs. moderate and severe HP.

NAFLD, nonalcoholic fatty liver disease; HP, hyperechoic pancreas; MS, metabolic syndrome; BMI, body mass index; SBP, systolic blood pressure; DBP, diastolic blood pressure; GLC, fasting blood glucose; HbA1c, plasma hemoglobin A1c; TG, triglyceride; $\mathrm{Cr}$, creatinine level; BUN, blood urea nitrogen; LDL, low-density lipoprotein; HDL, high-density lipoprotein.

The demographic characteristics and biochemical data stratified according to the four study subgroups are summarized in Table 2 (FP1 criterion) and Table 3 (FP2 criterion). Using the FP1 criterion, the prevalence of isolated HP (group 3) and all HP (groups 3 and 4) was $33.0 \%$ (458 of 1,386) and $36.9 \%$ (511 of 1,386), respectively. The prevalence of isolated NAFLD (group 2) and all NAFLD (group 2 and 4) was $1.4 \%(20$ of 1,386$)$ and $38.3 \%(531$ of 1,386$)$, respectively. Using the FP2 criterion, the prevalence of isolated HP (group 3) and all HP (groups 3 and 4) was $12.8 \%$ (178 of 1,386) and 27.8\% (386 of 1,386), respectively. The prevalence of isolated NAFLD (group 2 ) and all NAFLD (group 2 and 4) was 10.5\% (145 of 1,386) and $38.3 \%$ (531 of 1,386), respectively.

Using the FP1 and FP2 criteria, participants with isolated NAFLD had higher body weight, BMI, waist circumference, blood pressure, triglyceride levels, and cholesterol levels than those with isolated HP. However, participants with isolated HP showed higher fasting blood glucose levels and HbA1c values than those with isolated NAFLD. Among the participants with fatty pancreas, $52.7 \%$ (511 of 969) of
Table 4. Log-rank P-values of pairwise comparisons for the cumulative incidence of glycemic progression among the four study groups

\begin{tabular}{cccc}
\hline & Group 1 & Group 2 & Group 3 \\
\hline FP1 criterion & & & \\
Group 2 & $<0.001$ & & \\
Group 3 & $<0.001$ & 0.792 & \\
Group 4 & $<0.001$ & 0.143 & $<0.001$ \\
FP2 criterion & & & \\
Group 2 & $<0.001$ & & \\
Group 3 & 0.004 & 0.001 & $<0.001$ \\
Group 4 & $<0.001$ & $<0.001$ & \\
\hline
\end{tabular}

FP1 (fatty pancreas 1) criterion, normal vs. mild HP or more; FP2 (fatty pancreas 2) criterion, normal and mild HP vs. moderate and severe HP. Group 1 included those without NAFLD and HP; group 2, those with isolated NAFLD; group 3, those with isolated HP; group 4, those with both HP and NAFLD.

$H P$, hyperechoic pancreas; NAFLD, nonalcoholic fatty liver disease. 
those grouped using the FP1 criterion and $68.4 \%$ (386 of 564) of those grouped using the FP2 criterion showed concomitant NAFLD.

\section{Cumulative Incidence of Glycemic Progression}

Table 4 and Figs. 3 and 4 show the cumulative incidence of glycemic progression among the four subgroups according to the FP1 and FP2 criteria. The presence of NAFLD or HP showed cumulative aggravation of glycemic progression and was associated with a higher probability of glycemic progression in both criteria $(P<0.001$



NAFLD $531 \quad 531509 \quad 434 \quad 387 \quad 324 \quad 266 \quad 207 \quad 132 \quad 12 \quad 0$ No NAFLD $855854 \quad 833735 \quad 663583489409266 \quad 27 \quad 1$

A

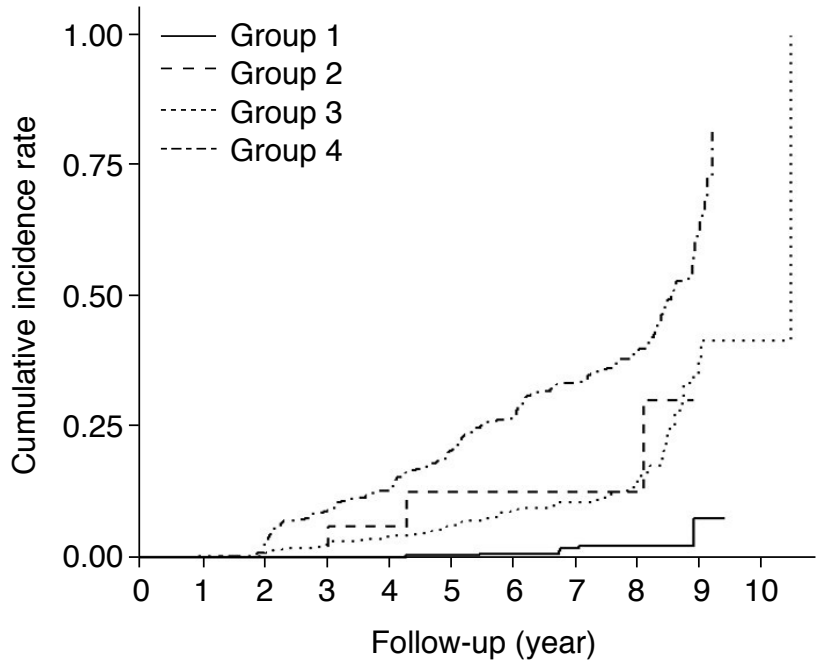

$\begin{array}{llllllllllll}\text { Group } 1 & 397 & 397 & 391 & 340 & 311 & 271 & 227 & 188 & 118 & 12 & 0\end{array}$ $\begin{array}{llllllllllll}\text { Group } 2 & 20 & 20 & 19 & 17 & 14 & 12 & 11 & 10 & 6 & 0 & 0\end{array}$ Group $3 \quad 458 \quad 457 \quad 442 \begin{array}{llllllllll}395 & 352 & 312 & 262 & 221 & 148 & 15 & 1\end{array}$ $\begin{array}{llllllllllll}\text { Group } 4 & 511 & 511 & 490 & 417 & 373 & 312 & 255 & 197 & 126 & 12 & 0\end{array}$ for both) (Figs. 3A, B, 4A). Using the FP1 criterion, although all subgroups showed cumulative aggravation for glycemic progression, no significant differences between groups 2 and $3(P=0.793)$ and between groups 2 and $4(P=0.141)$ were observed (Fig. 3C). Using the FP2 criterion, however, the probability of glycemic progression across the four subgroups showed cumulative aggravation for NAFLD and HP (all P<0.05) (Fig. 4B). Isolated HP showed a higher probability of glycemic progression than isolated NAFLD using the FP2 criterion $(P<0.001)$. The highest probability of glycemic

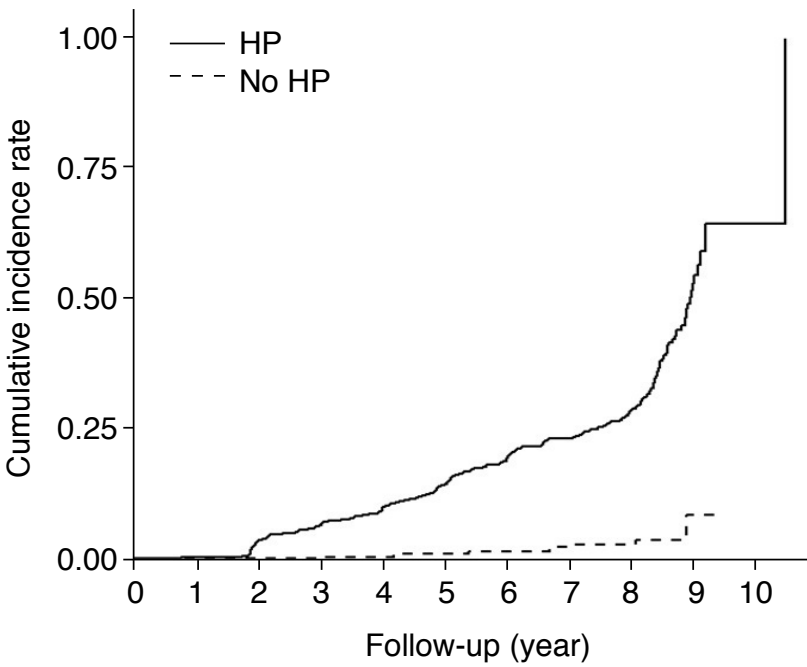

HP $969 \begin{array}{llllllllll}968 & 932 & 812 & 725 & 624 & 517 & 418 & 274 & 27 & 1\end{array}$


B

Fig. 3. The cumulative incidence of glycemic progression during follow-up.

A. Between participants with nonalcoholic fatty liver disease (NAFLD) and those without NAFLD are compared $(P<0.001)$. B, C. This is the result of cumulative incidence of glycemic progression during the follow-up using fatty pancreas 1 criterion. B. Between participants with hyperechoic pancreas (HP) and those without HP are compared $(\mathrm{P}<0.001)$. C. This is the result of the participants categorized into four study groups.

C 


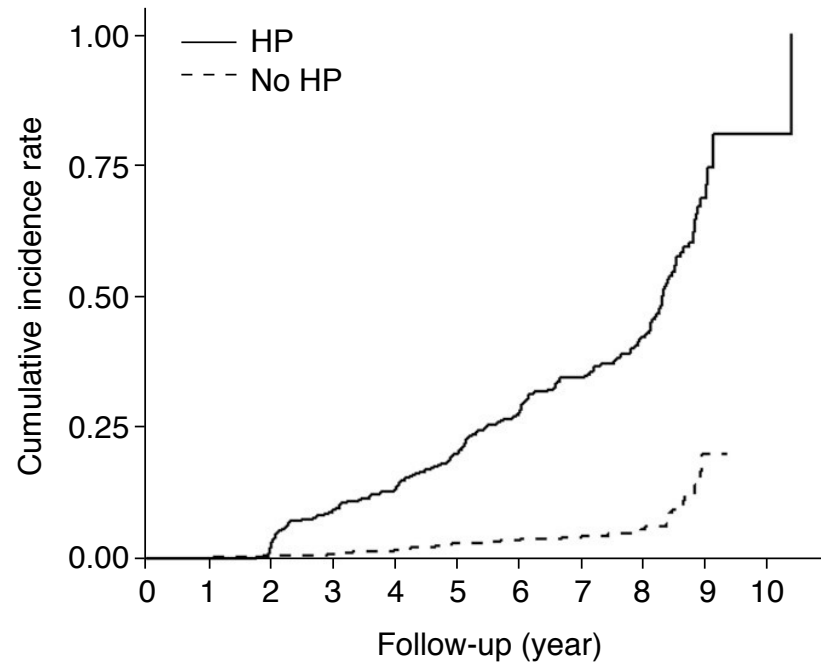

HP $\begin{array}{lllllllllll}564 & 564 & 543 & 467 & 418 & 350 & 279 & 220 & 142 & 16 & 1\end{array}$ No HP $822821799702632557476 \quad 396 \quad 256 \quad 23 \quad 0$

A

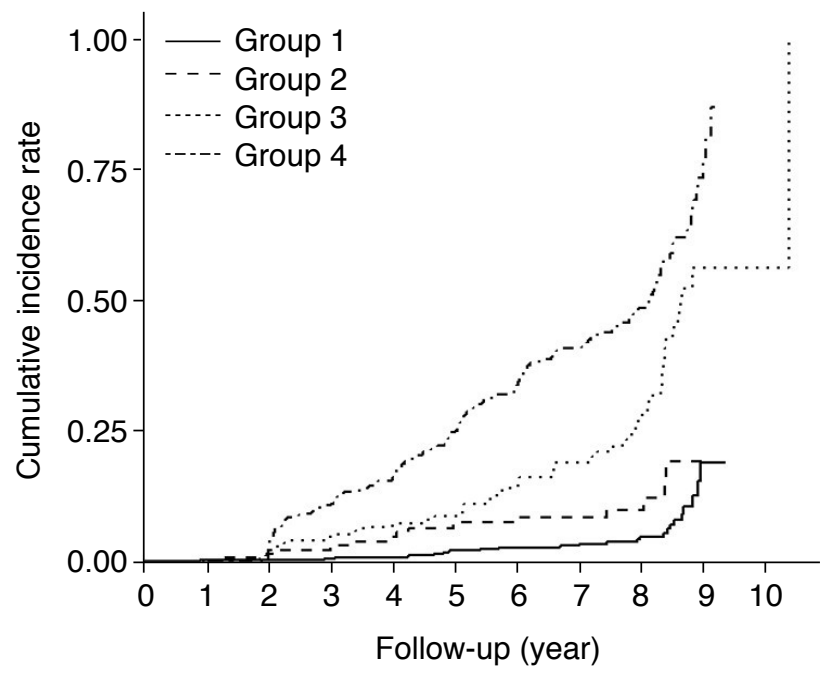

$\begin{array}{lrrrrrrrrrrr}\text { Group 1 } & 677 & 676 & 660 & 581 & 522 & 463 & 391 & 327 & 213 & 21 & 0 \\ \text { Group 2 } & 145 & 145 & 139 & 121 & 110 & 94 & 85 & 69 & 43 & 2 & 0 \\ \text { Group 3 } & 178 & 178 & 173 & 154 & 141 & 120 & 98 & 82 & 53 & 6 & 1 \\ \text { Group 4 } & 386 & 386 & 370 & 313 & 277 & 230 & 181 & 138 & 89 & 10 & 0 \\ \text { B } & & & & & & & & & & & \end{array}$

Fig. 4. The cumulative incidence of glycemic progression during follow-up using the fatty pancreas 2 criterion.

A. Between participants with hyperechoic pancreas $(H P)$ and those without HP are compared $(P<0.001) B$. This is the result of the participants categorized into four study groups.

progression was observed in the subgroup with both NAFLD and HP (group $4, \mathrm{P}<0.001$ ) using the FP2 criterion.

\section{Cox Proportional Hazard Regression Models}

Table 5 show the results of univariable analysis performed to evaluate the associations of NAFLD and HP with the probability of glycemic progression. Using the FP1 and FP2 criteria, the other three subgroups showed a significant difference from group 1, used as the reference for glycemic progression (all $P<0.05$ ). However, using the FP1 criterion with group 2 as the reference, no significant difference was identified in group $3(P=0.802)$ and group $4(P=0.124)$ for glycemic progression. Conversely, using the FP2 criterion, these three subgroups showed a significant difference in glycemic progression (all $\mathrm{P}<0.05$ ). In other words, isolated HP of more than a moderate degree (group 3) and the presence of both HP and NAFLD were associated with significantly higher probabilities of glycemic progression than isolated NAFLD (HR, 2.59; $P=0.002$ and HR, 5.26; $\mathrm{P}<0.001$, respectively).

In the univariable analysis using the FP2 criterion, to clarify which factor and group could best explain the association with glycemic progression, further analyses were performed by adding variables in five steps (Table 6, Supplementary Table 1). In the first step (model 1), each group (groups 1, 3, and 4), age, and sex were used as the independent variables. In model 2, weight, BMI, and waist
Table 5. Univariable analysis to evaluate factors associated with the probability of glycemic progression

\begin{tabular}{cccccc}
\hline & \multicolumn{2}{c}{ FP1 } & & \multicolumn{2}{c}{ FP2 } \\
\cline { 2 - 3 } & $\begin{array}{c}\text { Hazard ratio } \\
(95 \% \mathrm{Cl})\end{array}$ & P-value & & $\begin{array}{c}\text { Hazard ratio } \\
(95 \% \mathrm{Cl})\end{array}$ & P-value \\
\hline $\begin{array}{l}\text { Group 1 } \\
\text { (reference) }\end{array}$ & & & & & \\
Group 2 & $11.02(2.76-44.09)$ & $<0.001$ & $2.46(1.29-4.67)$ & 0.006 \\
Group 3 & $9.50(4.12-21.91)$ & $<0.001$ & $6.38(3.98-10.23)$ & $<0.001$ \\
Group 4 & $26.98(11.96-60.84)$ & $<0.001$ & $12.94(8.68-19.30)$ & $<0.001$
\end{tabular}

Group 2

(reference)

$\begin{array}{lllll}\text { Group } 1 & 0.09(0.02-0.36) & <0.001 & 0.41(0.21-0.77) & 0.006\end{array}$

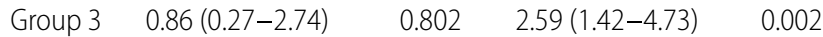

$\begin{array}{lllll}\text { Group } 4 & 2.45(0.78-7.66) & 0.124 & 5.26(3.05-9.08) & <0.001\end{array}$

FP1 (fatty pancreas 1) criterion, normal vs. mild HP or more; FP2 (fatty pancreas 2) criterion, normal and mild HP vs. moderate and severe HP. Group 1 included those without NAFLD and HP; group 2, those with isolated NAFLD; group 3, those with isolated HP; group 4, those with both HP and NAFLD.

$\mathrm{Cl}$, confidence interval; HP, hyperechoic pancreas; NAFLD, nonalcoholic fatty liver disease.

circumference were added to model 1. In model 3, triglyceride, low-density lipoprotein, high-density lipoprotein, and cholesterol, which are known factors related to fatty liver, were added to model 2. In model 4, plasma glucose and $\mathrm{HbA1c}$ were added to model 
Table 6. Multivariable analysis to evaluate factors associated with the probability of glycemic progression based on the FP2 criterion

\begin{tabular}{|c|c|c|}
\hline & Hazard ratio $(95 \% \mathrm{Cl})$ & P-value \\
\hline \multicolumn{3}{|c|}{ Group 1 (reference) } \\
\hline \multicolumn{3}{|l|}{ Model $1^{\text {a) }}$} \\
\hline Group 2 & $2.36(1.23-4.53)$ & 0.010 \\
\hline Group 3 & $5.73(3.53-9.30)$ & $<0.001$ \\
\hline Group 4 & $12.03(7.94-18.22)$ & $<0.001$ \\
\hline \multicolumn{3}{|l|}{ Model $2^{\text {b) }}$} \\
\hline Group 2 & $1.88(0.98-3.64)$ & 0.059 \\
\hline Group 3 & $4.58(2.79-7.50)$ & $<0.001$ \\
\hline Group 4 & $8.15(5.22-12.71)$ & $<0.001$ \\
\hline \multicolumn{3}{|l|}{ Model $3^{\text {c) }}$} \\
\hline Group 2 & $1.65(0.85-3.20)$ & 0.141 \\
\hline Group 3 & $4.46(2.72-7.34)$ & $<0.001$ \\
\hline Group 4 & $7.35(4.68-11.56)$ & $<0.001$ \\
\hline \multicolumn{3}{|l|}{ Model $4^{d)}$} \\
\hline Group 2 & $2.00(1.03-3.90)$ & 0.042 \\
\hline Group 3 & $3.93(2.39-6.46)$ & $<0.001$ \\
\hline Group 4 & $5.98(3.78-9.47)$ & $<0.001$ \\
\hline \multicolumn{3}{|l|}{ Model $5^{\mathrm{e})}$} \\
\hline Group 2 & $2.09(1.07-4.08)$ & 0.031 \\
\hline Group 3 & $3.87(2.34-6.40)$ & $<0.001$ \\
\hline Group 4 & $5.85(3.67-9.31)$ & $<0.001$ \\
\hline \multicolumn{3}{|c|}{ Group 2 (reference) } \\
\hline \multicolumn{3}{|l|}{ Model $1^{\text {a) }}$} \\
\hline Group 1 & $0.42(0.22-0.81)$ & 0.010 \\
\hline Group 3 & $2.42(1.32-4.44)$ & 0.004 \\
\hline Group 4 & $5.09(2.95-8.79)$ & $<0.001$ \\
\hline \multicolumn{3}{|l|}{ Model $2^{b)}$} \\
\hline Group 1 & $0.53(0.28-1.02)$ & 0.059 \\
\hline Group 3 & $2.43(1.32-4.45)$ & 0.004 \\
\hline Group 4 & $4.32(2.49-7.50)$ & $<0.001$ \\
\hline \multicolumn{3}{|l|}{ Model $3^{c}$} \\
\hline Group 1 & $0.61(0.31-1.18)$ & 0.141 \\
\hline Group 3 & $2.71(1.47-4.99)$ & 0.001 \\
\hline Group 4 & $4.46(2.57-7.74)$ & $<0.001$ \\
\hline \multicolumn{3}{|l|}{ Model $4^{\mathrm{d})}$} \\
\hline Group 1 & $0.50(0.26-0.97)$ & 0.042 \\
\hline Group 3 & $1.96(1.05-3.66)$ & 0.034 \\
\hline Group 4 & $2.99(1.71-5.24)$ & $<0.001$ \\
\hline
\end{tabular}

Table 6. Continued

\begin{tabular}{rcr}
\hline & Hazard ratio $(95 \% \mathrm{Cl})$ & P-value \\
\hline Model 5 $^{\mathrm{e}}$ & & \\
Group 1 & $0.48(0.25-0.94)$ & 0.031 \\
Group 3 & $1.85(0.99-3.45)$ & 0.053 \\
Group 4 & $2.80(1.59-4.92)$ & $<0.001$
\end{tabular}

FP2 (fatty pancreas 2) criterion, normal and mild HP vs. moderate and severe HP. $\mathrm{Cl}$, confidence interval; HP, hyperechoic pancreas; BMI, body mass index; LDL, lowdensity lipoprotein; HDL, high-density lipoprotein; HbA1C, hemoglobin A1C.

${ }^{a)}$ Adjusted for each group, age and sex. ${ }^{b}$ Adjusted for each group, age, sex, weight, BMI, and waist circumference. 'Adjusted for each group, age, sex, weight, BMI, waist circumference, triglycerides, LDL, HDL, and total cholesterol. ${ }^{\text {d) Adjusted for }}$ each group, age, sex, weight, BMI, waist circumference, triglycerides, LDL, HDL, total cholesterol, fasting blood glucose, and HbA1c. ${ }^{\text {e) }}$ Fully adjusted model, adjusted for each group, age, sex, weight, BMI, waist circumference, systolic blood pressure, diastolic blood pressure, triglycerides, creatinine, blood urea nitrogen, LDL, HDL, total cholesterol, fasting blood glucose, and HbA1c.

3. Model 5 was a fully adjusted model. The multivariable survival analysis found that isolated HP (group 3) and combined HP and NAFLD (group 4) had the highest positive associations with glycemic progression in models 1, 2, and 3. Even after adjusting for glycemic parameters, the groups showed a significantly higher probability of glycemic progression than group 2 as a reference. In the fully adjusted model, group 4 revealed a significantly higher probability of glycemic progression than $\mathrm{HbA} 1 \mathrm{c}$.

\section{Effect of HP Severity on Glycemic Progression}

The rate of glycemic progression according to the severity of HP was as follows: $8.1 \%$ (33 of 405 ) in patients with mild HP, 28.3\% (104 of 368) in patients with moderate HP, and $59.2 \%$ (116 of 196) in patients with severe HP. To identify the relationship of glycemic progression according to the severity of HP on US, a Cox proportional hazard model was used. On univariable survival analysis, HP of all degrees and all clinical variables, except creatinine, were significantly correlated with glycemic progression $(P<0.05)$. In the fully adjusted model, the HR for glycemic progression increased with the severity of $H P(H R, 2.718 ; P=0.009$ in mild HP; HR, 6.365; $\mathrm{P}<0.001$ in moderate HP; and HR, 8.984; $\mathrm{P}<0.001$ in severe HP) (Supplementary Table 2). Additionally, NAFLD, fasting glucose, $\mathrm{HbA1c}$, and creatinine were also significant predictors of glycemic progression $(\mathrm{P}<0.05)$.

\section{Discussion}

Our study showed that the presence of NAFLD or HP was associated with a high probability of glycemic progression. Further, an independent association was identified between HP and glycemic 
progression independent of NAFLD during the follow-up. In particular, isolated HP of a moderate or higher degree was more highly associated with glycemic progression than isolated NAFLD (HR, 2.59; $P=0.002)$. Additionally, the presence of both HP and NAFLD (FP2, group 4) showed the most significant association with glycemic progression independent of clinical parameters. Although isolated HP of a mild or higher degree (FP1) and isolated NAFLD were also associated with an increased probability of glycemic progression, there was no significant difference between the subgroups.

In this study, we divided the cutoff value of HP into mild and moderate degrees. The normal echogenicity of the pancreas is equal to or slightly greater than that of the liver $[22,23]$. However, in many previous studies, fatty pancreas was simply defined as higher echogenicity than that of the liver or renal cortex on US or endoscopic US. This diagnostic criterion for fatty pancreas is slightly vague and can occasionally be challenging with respect to the possible concurrence of NAFLD. The prevalence of fatty pancreas in previous studies varied widely, from $8.5 \%$ to $61.4 \%[6,17,24-28]$, probably owing to the lack of a widely accepted imaging modality or a cutoff point for diagnosing fatty pancreas. In this study, the prevalence of fatty pancreas was $69.9 \%$ using the FP1 criterion and $40.6 \%$ using the FP2 criterion, and this wide discrepancy was attributed to mild fatty pancreas (29.3\%). Therefore, for an accurate diagnosis of fatty pancreas, we believe that a similar or mildly lower echogenicity compared with the retroperitoneal fat or a definite increase in echogenicity compared with the renal cortex can be suitable diagnostic criteria.

In humans, pancreatic fat deposition has a close association with insulin resistance, NAFLD, increased BMI, and metabolic syndrome $[27,29]$. Many previous studies have indicated that diabetes is independently associated with fatty pancreas and vice versa. Fat deposition in the pancreas may contribute to a loss of $\beta$-cell mass and function, which can lead to the development of diabetes $[27,30]$. Conversely, as a consequence of insulin resistance, $\beta$-cell apoptosis occurs with replacement by adipose tissue in the pancreas. In this study, we demonstrated that isolated HP was independently associated with glycemic progression, which is in close agreement with the result of Hung et al. [17].

Furthermore, obesity also leads to fat infiltration in the pancreas related to circulating free fatty acids, which also drive the development of NAFLD. A strong association between fatty pancreas and NAFLD has been reported in several studies $[20,24,25,31,32]$, and the prevalence of concurrent NAFLD with fatty pancreas on US has been reported to be up to $67.9 \%$ [8]. This concomitant phenomenon is attributed to the same possible pathogenesis [33]. Similarly, a considerable number of participants with fatty pancreas in our study had concomitant NAFLD $(52.7 \%$ and $68.4 \%$ using the FP1 and FP2 criteria, respectively). To identify the isolated effect of fatty pancreas on glycemic progression, we divided the study group into four subgroups. Although isolated NAFLD and isolated HP were both strongly associated with glycemic progression in our study and in a previous study [17], there was no significant difference regarding glycemic progression between the groups using the FP1 criterion in our study and in a previous study [17]. However, in our study, isolated HP of a moderate or higher degree showed a more significant association with glycemic progression than isolated NAFLD. This indirectly suggests that a moderate or higher degree of fatty pancreas can be considered a predictor of glycemic progression. In this respect, these findings are different from those of the previous study.

In the present study, participants with both NAFLD and HP (group 4, both the FP1 and FP2 criteria) showed the highest glycemic progression risk. Some researchers have shown a synergistic effect on glycemic progression $[33,34]$. Individuals with nonalcoholic steatohepatitis and fatty pancreas had a higher prevalence of prediabetes and diabetes as well as higher glucose parameters than those with nonalcoholic steatohepatitis alone. As shown in the above study, it is believed that fatty liver and fatty pancreas may influence pancreas islet cell function through mechanisms that act together or separately. A study of the effects of fatty pancreas on the pathogenesis of diabetes is still under way; however, studies on the effects of pancreas islet function coexisting with fatty liver are also needed.

In this study, the risk for glycemic progression significantly increased with the severity of HP independent of NAFLD or other clinical parameters (HR, 2.718 in mild HP; 6.365 in moderate HP; and 8.984 in severe HP). Although there is convincing evidence that fatty pancreas increases the risk of diabetes, no studies have evaluated glycemic progression according to the severity of HP. In studies on NAFLD, the incidence rate of diabetes progressively increased according to the severity of NAFLD on US, and more severe NAFLD was related to a higher risk of glycemic progression $[35,36]$. On the basis of the similar pathogenesis of fatty pancreas and NAFLD, we speculate that our results could be similar to those of studies on NAFLD. However, further validated analyses are needed with more participants.

This study had a few limitations. First, the prevalence of fatty pancreas was higher (69.9\% using the FP1 criterion and $40.6 \%$ using the FP2 criterion) than has been reported in previous studies. However, as mentioned above, its prevalence has been reported to vary widely $(8.5 \%-61.4 \%)$, which can be attributed to ambiguous diagnostic criteria and insufficient evidence for the severity of fatty pancreas. We believe that the prevalence of fatty pancreas in our 
study is acceptable and that a large sample size would be beneficial for generalization to a larger population of interest. Further studies are warranted to clarify the effects of fatty pancreas. Second, our study included a small number of patients with isolated NAFLD using the FP1 criterion. We speculate that a reason for this could be the considerable number of participants with concurrent NAFLD with mild HP (30.9\%, 125 of 405$)$. Additionally, the number of patients with diabetes was relatively very small. Because our study included only participants who attended health screening evaluations, these patients can be followed up at the department of endocrinology. Therefore, further studies with a larger number of participants are needed to validate our results. Third, this was single-center study on an Asian population and the follow-up period was relatively short (2.0-10.4 years). The follow-up period may not have been sufficient to evaluate the association between fatty pancreas and glycemic progression. Therefore, similar studies with a longer follow-up period are needed to verify our results in other populations. Fourth, as this study was conducted on participants who underwent health screening, the study sample is not fully representative of the general healthy population. Fifth, our study did not use the homeostatic model assessment of insulin resistance to evaluate insulin resistance because it is not routine laboratory data for health screening participants in our hospital. However, several previous studies $[37,38]$ alternatively assessed fasting glucose levels or $\mathrm{HbA} 1 \mathrm{c}$ as markers of insulin resistance. Therefore, we believe our results can be considered acceptable. Sixth, interobserver and intraobserver variability as well as variability of US measurements with different machines might have affected the overall outcomes. Although the interobserver agreement of fatty pancreas on US in our study was similar to that reported by a previous study [10], with substantial agreement ( $\mathrm{K}=0.78$ vs. $\mathrm{K}=0.63$ ), research on intraobserver variability and variability across different US machines is insufficient. Therefore, further research is required to validate our results. Finally, we had no data on the histologic evaluation of the pancreas. However, a pancreatic biopsy is very invasive and, therefore, not suitable for practice in healthy participants. Instead, this study used one of the best noninvasive methods for evaluating fat in the liver and pancreas, which has been proven in previous studies to be a reliable, reproducible, and noninvasive screening tool for fatty pancreas $[20,27,39,40]$

In conclusion, an increasing severity of HP on US is significantly correlated with glycemic progression. Moreover, isolated HP of more than moderate severity is a relevant factor for predicting glycemic progression independent of NAFLD. Therefore, the severity of pancreatic echogenicity, particularly a more than moderate degree, can be considered as an early predictor of glycemic progression and could have potential benefits in clinical practice.
ORCID: Jiyun Oh: https://orcid.org/0000-0002-9452-1805; Hyun Jeong Park: https:// orcid.org/0000-0003-1701-0478; Eun Sun Lee: https://orcid.org/0000-0003-07807985; Sung Bin Park: https://orcid.org/0000-0002-4155-9260; Byung Ihn Choi: https://orcid.org/0000-0002-5613-1881; Soohyun Ahn: https://orcid.org/0000-00015016-5469

\section{Author Contributions}

Conceptualization: Oh J, Park HJ, Lee ES, Park SB, Choi BI. Data acquisition: Oh J, Park HJ, Ahn S. Data analysis or interpretation: Oh J, Park HJ, Ahn S. Drafting of the manuscript: Oh J, Park HJ, Ahn S. Critical revision of the manuscript: Oh J, Park HJ, Lee ES, Park SB, Choi BI, Ahn S. Approval of the final version of the manuscript: all authors.

\section{Conflict of Interest}

Byung Ihn Choi serves as Editor for the Ultrasonography, but have no role in the decision to publish this article. All remaining authors have declared no conflicts of interest.

\section{Acknowledgments}

This Research was supported by a Chung-Ang University research grant in 2019.

\section{Supplementary Material}

Supplementary Table 1. Univariable and multivariable analysis to evaluate associated factors with probability of glycemic progression based on FP2 criterion (https://doi.org/10.14366/usg.20122).

Supplementary Table 2. Univariable and multivariable analysis for HP grading effect to glycemic progression using Cox proportional hazard model (https://doi.org/10.14366/usg.20122).

\section{References}

1. Anstee QM, Targher G, Day CP. Progression of NAFLD to diabetes mellitus, cardiovascular disease or cirrhosis. Nat Rev Gastroenterol Hepatol 2013;10:330-344.

2. Chang Y, Jung HS, Yun KE, Cho J, Cho YK, Ryu S. Cohort study of non-alcoholic fatty liver disease, NAFLD fibrosis score, and the risk of incident diabetes in a Korean population. Am J Gastroenterol 2013; 108:1861-1868.

3. Hamaguchi M, Kojima T, Takeda N, Nagata C, Takeda J, Sarui $\mathrm{H}$, et al. Nonalcoholic fatty liver disease is a novel predictor of cardiovascular disease. World J Gastroenterol 2007;13:1579-1584.

4. Kotronen A, Yki-Jarvinen H. Fatty liver: a novel component of the metabolic syndrome. Arterioscler Thromb Vasc Biol 2008;28:27-38.

5. Catanzaro R, Cuffari B, Italia A, Marotta F. Exploring the metabolic syndrome: nonalcoholic fatty pancreas disease. World J 
Gastroenterol 2016;22:7660-7675.

6. Choi CW, Kim GH, Kang DH, Kim HW, Kim DU, Heo J, et al. Associated factors for a hyperechogenic pancreas on endoscopic ultrasound. World J Gastroenterol 2010;16:4329-4334.

7. Dite $P$, Blaho M, Bojkova M, Jabandziev P, Kunovsky L. Nonalcoholic fatty pancreas disease: clinical consequences. Dig Dis 2020;38:143149.

8. Lee JS, Kim SH, Jun DW, Han JH, Jang EC, Park JY, et al. Clinical implications of fatty pancreas: correlations between fatty pancreas and metabolic syndrome. World J Gastroenterol 2009;15:18691875.

9. Obora A, Kojima T, Kato T, Endo M, Miyawaki K, Fukuta N, et al. Associations between bright pancreas and features of metabolic syndrome. Ningen Dock 2012;26:935-943.

10. Ou HY, Wang CY, Yang YC, Chen MF, Chang CJ. The association between nonalcoholic fatty pancreas disease and diabetes. PLoS One 2013;8:e62561.

11. Tariq H, Nayudu S, Akella S, Glandt M, Chilimuri S. Non-alcoholic fatty pancreatic disease: a review of literature. Gastroenterology Res 2016;9:87-91.

12. Quinn SF, Gosink BB. Characteristic sonographic signs of hepatic fatty infiltration. AJR Am J Roentgenol 1985; 145:753-755.

13. Worthen NJ, Beabeau D. Normal pancreatic echogenicity: relation to age and body fat. AJR Am J Roentgenol 1982;139:1095-1098.

14. Smits MM, van Geenen EJ. The clinical significance of pancreatic steatosis. Nat Rev Gastroenterol Hepatol 2011;8:169-177.

15. Marks WM, Filly RA, Callen PW. Ultrasonic evaluation of normal pancreatic echogenicity and its relationship to fat deposition. Radiology 1980;137:475-479.

16. Makino N, Shirahata N, Honda T, Ando Y, Matsuda A, Ikeda Y, et al. Pancreatic hyperechogenicity associated with hypoadiponectinemia and insulin resistance: a Japanese population study. World J Hepatol 2016;8:1452-1458.

17. Hung CS, Tseng PH, Tu CH, Chen CC, Liao WC, Lee YC, et al. Increased pancreatic echogenicity with US: relationship to glycemic progression and incident diabetes. Radiology 2018;287:853-863.

18. Al-Haddad M, Khashab M, Zyromski N, Pungpapong S, Wallace MB, Scolapio J, et al. Risk factors for hyperechogenic pancreas on endoscopic ultrasound: a case-control study. Pancreas 2009;38:672-675.

19. Garcia TS, Rech TH, Leitao CB. Pancreatic size and fat content in diabetes: a systematic review and meta-analysis of imaging studies. PLoS One 2017;12:e0180911.

20. Lesmana CR, Pakasi LS, Inggriani S, Aidawati ML, Lesmana LA. Prevalence of non-alcoholic fatty pancreas disease (NAFPD) and its risk factors among adult medical check-up patients in a private hospital: a large cross sectional study. BMC Gastroenterol 2015;15:174.

21 Needleman L, Kurtz AB, Rifkin MD, Cooper HS, Pasto ME, Goldberg
BB. Sonography of diffuse benign liver disease: accuracy of pattern recognition and grading. AJR Am J Roentgenol 1986;146:10111015.

22. Ghorashi B, Rector WR. Gray scale sonographic anatomy of the pancreas. J Clin Ultrasound 1977;5:25-29.

23. Hancke S. Ultrasonic scanning of the pancreas. J Clin Ultrasound 1976;4:223-230.

24. Sepe PS, Ohri A, Sanaka S, Berzin TM, Sekhon S, Bennett G, et al. A prospective evaluation of fatty pancreas by using EUS. Gastrointest Endosc 2011;73:987-993.

25. Wong VW, Wong GL, Yeung DK, Abrigo JM, Kong AP, Chan RS, et al. Fatty pancreas, insulin resistance, and beta-cell function: a population study using fat-water magnetic resonance imaging. Am J Gastroenterol 2014;109:589-597.

26. Sotoudehmanesh R, Tahmasbi A, Sadeghi A, Hosseini H, Mohamadnejad $\mathrm{M}$. The prevalence of nonalcoholic fatty pancreas by endoscopic ultrasonography. Pancreas 2019;48:1220-1224.

27. Wang CY, Ou HY, Chen MF, Chang TC, Chang CJ. Enigmatic ectopic fat: prevalence of nonalcoholic fatty pancreas disease and its associated factors in a Chinese population. J Am Heart Assoc 2014;3:e000297.

28. Weng S, Zhou J, Chen X, Sun Y, Mao Z, Chai K. Prevalence and factors associated with nonalcoholic fatty pancreas disease and its severity in China. Medicine (Baltimore) 2018;97:e11293.

29. Roever L. Pancreatic steatosis: is it related to obesity, diabetes mellitus and metabolic syndrome. Pancreat Disord Ther 2015;5:3.

30. Begovatz P, Koliaki C, Weber K, Strassburger K, Nowotny B, Nowotny $P$, et al. Pancreatic adipose tissue infiltration, parenchymal steatosis and beta cell function in humans. Diabetologia 2015;58:1646-1655.

31. van Geenen EJ, Smits MM, Schreuder TC, van der Peet DL, Bloemena E, Mulder CJ. Nonalcoholic fatty liver disease is related to nonalcoholic fatty pancreas disease. Pancreas 2010;39:11851190.

32. Lesmana CR, Gani RA, Lesmana LA. Non-alcoholic fatty pancreas disease, pancreatic cancer, and impact of endoscopic ultrasound examination on screening and surveillance. EMJ Gastroenterol 2017;6:69-74.

33. Gerst F, Wagner R, Kaiser G, Panse M, Heni M, Machann J, et al. Metabolic crosstalk between fatty pancreas and fatty liver: effects on local inflammation and insulin secretion. Diabetologia 2017;60:2240-2251.

34. Della Corte C, Mosca A, Majo F, Lucidi V, Panera N, Giglioni E, et al. Nonalcoholic fatty pancreas disease and nonalcoholic fatty liver disease: more than ectopic fat. Clin Endocrinol (Oxf) 2015;83:656662.

35. Park SK, Seo MH, Shin HC, Ryoo JH. Clinical availability of nonalcoholic fatty liver disease as an early predictor of type 2 diabetes mellitus in Korean men: 5-year prospective cohort study. 
Hepatology 2013;57:1378-1383.

36. Byrne CD, Targher G. NAFLD: a multisystem disease. J Hepatol 2015;62(1 Suppl):S47-S64.

37. Saha S, Schwarz PE. Impact of glycated hemoglobin (HbA1C) on identifying insulin resistance among apparently healthy individuals. J Public Health 2017;25:505-512.

38. Shan $S$, Gu L, Lou Q, Ouyang X, Yu Y, Wu H, et al. Evaluation of glycemic control in patients with type 2 diabetes mellitus in Chinese communities: a cross-sectional study. Clin Exp Med 2017;17:79-84.

39. Uygun A, Kadayifci A, Demirci H, Saglam M, Sakin YS, Ozturk K, et al. The effect of fatty pancreas on serum glucose parameters in patients with nonalcoholic steatohepatitis. Eur J Intern Med 2015;26:37-41.

40. Zhou J, Li ML, Zhang DD, Lin HY, Dai XH, Sun XL, et al. The correlation between pancreatic steatosis and metabolic syndrome in a Chinese population. Pancreatology 2016;16:578-583. 